\title{
Advanced Processing of Micropatterned Elasto-Magnetic Membranes
}

\author{
Elizabeth L. Martin ${ }^{1}$, Matthew T. Bryan ${ }^{1}$, Stefano Pagliara ${ }^{2}$ and Feodor. Y. Ogrin ${ }^{1}$ \\ ${ }^{1}$ College of Engineering, Mathematics and Physical Sciences, University of Exeter, Exeter, EX4 4QL, UK. \\ ${ }^{2}$ Biosciences and Living Systems Institute, University of Exeter, Exeter, EX4 4QD, UK.
}

\begin{abstract}
We report on a fabrication of a highly structured elasto-magnetic membrane with microscopic unit cells using lithographic techniques and electrodeposition. The membrane comprises of micro-magnets of CoNiP and Co connected via an elastic PDMS network with an area spanning $12 \mathrm{~mm}^{2}$. Ellipsoidal CoNiP and circular Co particles were produced by electrodeposition to achieve diameters of $30 \mu \mathrm{m}$ (major) and $10 \mu \mathrm{m}$ (minor), and $10 \mu \mathrm{m}$ respectively, with a thickness in the range of 6-10 $\mu \mathrm{m}$. The electrodeposition parameters have been optimized on deposition of CoNiP films to produce an in-plane coercivity for CoNiP elements of 435 Oe. The mechanical properties of the elasto-magnetic membrane were confirmed on application of an alternating magnetic field. The proposed methodology offers a new way for fabrication of novel microfluidic and MEMs devices.
\end{abstract}

Index Terms - CoNiP, Electrodeposition, MEMs, micro-magnets, PDMS,

\section{INTRODUCTION}

$\mathrm{M}$ icro-magnetic devices have been always a subject of wide interest due to their vast potential in medical and biotechnological applications, primarily due to their ability to be externally controlled with an applied magnetic field. However, the fabrication of structured magnetic materials as well as their integration with other materials at a microscale offers a number of challenges. Furthermore, the fabrication of anisotropically "hard" micro-magnets adds another order of complexity, whilst providing an extra value in applications in which a maximum mechanical torque is required. This has been seen as particularly important for the development in the area of Micro-Electro-Mechanical-Systems (MEMS). So far, there have been a number of micromachining techniques trialed for producing micro-magnets. This includes direct laser printing [1], micro-assembly [2] and electrodeposition [3][4]. Electrodeposition has been found to be a more popular and more accessible way to create magnetic microstructures, as it is easily integrated with lithographic methods and allows large volumes of materials to be deposited. However, compared to thin film deposition techniques or sintering methods used for bulk materials, the remaining challenge of electrodeposition is the lack of anisotropy that could provide large coercive fields in excess of several kG. As well as improving strength of the magnets at microscale, another challenge is related to the ways of integrating them with other materials in order to achieve the required functionality. For example, in MEMS this often includes elastic materials, which provide the capability of micromechanical actions, resulting from the combination of magnetic and elastic forces.

One particular area of interest where such systems are expected to find a range of applications is microfluidics. There is a growing need for active microscopic devices that could be integrated as part of the standard microfluidic chip

Manuscript received November 4, 2018; accepted December 10, 2018

Corresponding author: E. L. Martin (e-mail: em392@exeter.ac.uk).

Digital Object Identifier 10.1109/TMAG.2018.2887347 environment. This would enable a range of functionalities, such as pumping, stirring and valving, which are otherwise impossible at this scale of the fluidic geometry. Generally, due to the reduced dimensionality, any device has to obey the mechanics of Stokes regime in order to produce any useful motion [5]. This problem was succinctly formulated [6] and is being addressed theoretically [7], [8] and experimentally [9], [10], [11] by a number of research groups. In our own research effort, an investigation based on magnetic materials, has led to a concept of a ferromagnetic swimmer [12], [13]. Its design utilizes two magnetic elements, anisotropically soft and hard respectively, joined with an elastic link. Once activated with an oscillating magnetic field, the ferromagnetic swimmer is able to swim in liquid or pump fluids at a low Reynolds number regime. The devices have been successfully demonstrated experimentally [14], [15], however, the designs were implemented at a millimeter scale with the magnetic elements constructed using bulk materials (e.g. $\mathrm{NdFeB}$ magnets).

In this work, we are taking another step in developing microscopic ferromagnetic devices and focus on the challenge of integrating ferromagnetic compounds at a microscale. Firstly, we consider compounds demonstrating reasonably high coercive fields and investigate their properties with various parameters of electrodeposition. Secondly, we demonstrate a lithographic procedure that can be used to fabricate elastic structures with embedded ferromagnetic particles produced by electrodeposition. Finally, based on the concept of a ferromagnetic swimmer, we develop a twodimensional structured elasto-magnetic material that can be potentially used in microfluidic applications rendering functionality at low Reynolds number.

\section{Membrane Design}

The design of the proposed material is based on the geometry of a ferromagnetic swimmer detailed in [12], [15]. The single cell of the material contains three pairs of hard and soft ferromagnetic particles linked with elastic beams, as 


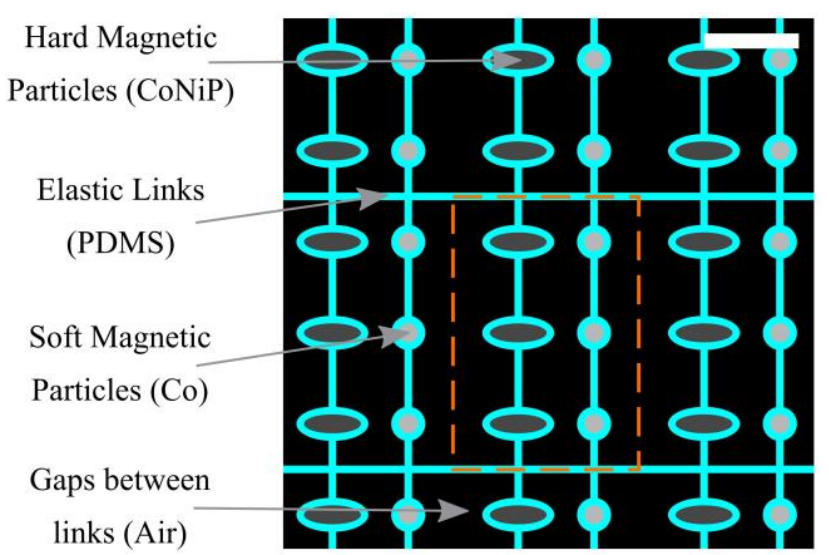

Fig. 1. Schematic of the elasto-magnetic membrane design (magnetic elements drawn to scale with the elastic links). The scale bar at the topright of the diagram indicates $50 \mu \mathrm{m}$. A unit cell (dotted) is comprised of three pairs of ferromagnetic swimmers.

shown in Fig. 1. Both the hard and soft ferromagnetic elements were produced by electrodeposition, using CoNiP and Co enriched solutions respectively [16], [17]. The elastic enclosure, together with the links, was formed on the basis of a silicon-based organic elastomer polydimethylsiloxane (PDMS). The magnetic elements were arranged in an alternating array of CoNiP and Co elements horizontally with vertical elements being of the same material, as shown in Fig. 1. The feature size of the design is in the tens of micron range with typical thicknesses of around 6-10 $\mu \mathrm{m}$. Here we demonstrate an example with the following dimensions (as shown on Fig. 1). The size of the ellipsoidal CoNiP element was $30 \mu \mathrm{m}$ in the major axis and $10 \mu \mathrm{m}$ in the minor axis. The circular Co elements have a diameter of $10 \mu \mathrm{m}$. The width of the links around the elements and connecting elements together were $4 \mu \mathrm{m}$. The dimensions of this unit cell: $98 \mu \mathrm{m}$ horizontally and $144 \mu \mathrm{m}$ vertically. The overall size of the membrane was $4 \mathrm{~mm} \times 4 \mathrm{~mm}$, attached to a frame with a 2 $\mathrm{mm}$ diameter circular central area.

\section{ELECTRODEPOSITION}

As stated above, both types of anisotropic magnetic elements were produced using electrodeposition. The main requirement in this case is that the element should provide a sufficient total magnetic moment, but with a minimal hysteresis to an applied magnetic field. To evaluate these properties a vibrating-sample-magnetometer (VSM) has been used.

As described above, the main challenge of this work was to find a material that would be possible to deposit with electrodeposition, to achieve microscopic dimensions but to maintain high anisotropy. This is very often lost due to the polycrystalline nature of compounds deposited with this method. A number of groups have attempted a similar research effort elsewhere and examined different candidates, including compounds such as $\mathrm{NdFeB}$ and $\mathrm{SmCo}$, which can provide coercive fields above $10 \mathrm{kG}$ and higher in its bulk form [18], [19]. However, to date there is still no compelling results demonstrating similar performance from equivalent materials produced by electrodeposition. Here we focus on CoNiP that previously has shown a good promise for thin film technologies [9][20][21] and we show that with the right conditions of deposition it can also be used for microscale applications.

As has been reported previously, cobalt-based alloys are good candidates to provide hard magnetic properties. Adding a non-magnetic element to a magnetic alloy can increase the coercivity of the material. It has been shown by Luborsky, through experiments on electrodepositing CoNi that $\mathrm{P}$ is the most effective of Group VA and VIB elements to increase the coercivity of the material, although, they are all capable of increasing the coercivity [20]. This is thought to be due to the segregation of phosphorous at the grain boundaries [22], which creates pinning sites for the domain walls when a magnetic field is applied.

The aqueous solution used to deposit the CoNiP was made of $33 \mathrm{~g} / \mathrm{L}$ of cobalt (II) sulphate heptahydrate $\left(\mathrm{CoSO}_{4} \cdot 7 \mathrm{H}_{2} \mathrm{O}\right)$, $60 \mathrm{~g} / \mathrm{L}$ of nickel (II) sulphate hexahydrate $\left(\mathrm{NiSO}_{4} \cdot 6 \mathrm{H}_{2} \mathrm{O}\right), 3.9$ $\mathrm{g} / \mathrm{L}$ of sodium hypophosphite monohydrate $\left(\mathrm{NaPO}_{2} \mathrm{H}_{2} \cdot \mathrm{H}_{2} \mathrm{O}\right)$ and $60 \mathrm{~g} / \mathrm{L}$ of ammonium chloride $\left(\mathrm{NH}_{4} \mathrm{Cl}\right),(\mathrm{pH} 5)$ [17]. Electrodeposition was performed in a cell with a Teflon base, a platinum mesh anode, a brass rod acting as a reference wire and a cathode made from a copper frame to which the samples were connected.

Electroplating parameters of CoNiP were first explored by depositing thin films $(\sim 1 \mu \mathrm{m}$ thick) of CoNiP onto thick (35 $\mu \mathrm{m})$ copper substrates. The substrates were all electrodeposited for the same time period (120 s), but the applied voltage was altered to change the current density. Average current density was found by measuring the average current during the deposition and dividing by the surface area that was electrodeposition. Figure 2(a) shows that the coercivity varies with a generally exponentially decreasing trend apart from at low current densities. Typically, low current densities produce hard magnets, whereas large current densities resulted in soft magnetic properties [Fig. 2(a)]. We have also investigated the dependence on temperature, which only had a minor effect on coercivity except for the softening effect at the highest temperatures and lowest current densities. The largest coercive field (1225 Oe) was produced at a temperature of $30^{\circ} \mathrm{C}$ with an average current density of 13.8 $\mathrm{mAcm}^{-2}$.

To examine the effect of depositing on different substrates, we investigated whether deposition on to gold-coated silicon affected the relationship between average current density and coercive field. While the overall trend appeared to be similar to that of the copper substrates, deposition onto silicon produced lower coercivites [Fig. 2(a)], with a maximum coercive field of $625 \mathrm{Oe}$. It is likely that this reduction in coercivity is due to the lower surface roughness of silicon [23].

In order to generate dipolar interactions between magnetic elements, the elasto-magnetic membranes require soft particles to be electrodeposited as well as hard particles. The soft elements were implemented based on a Co solution, with the 


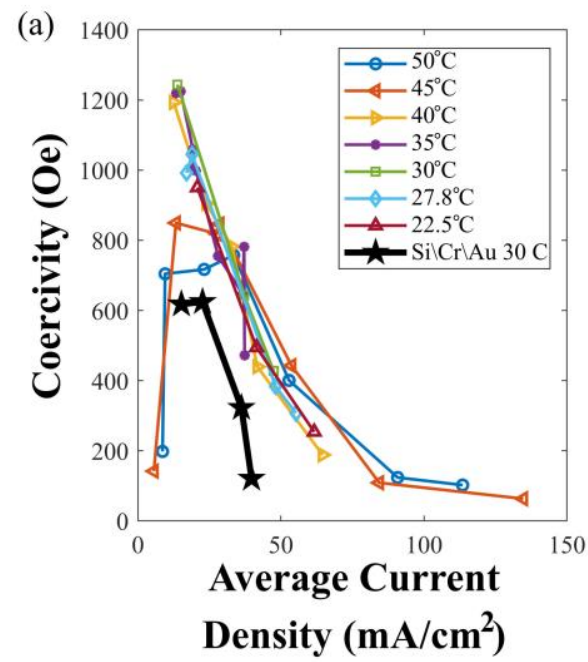

(b)

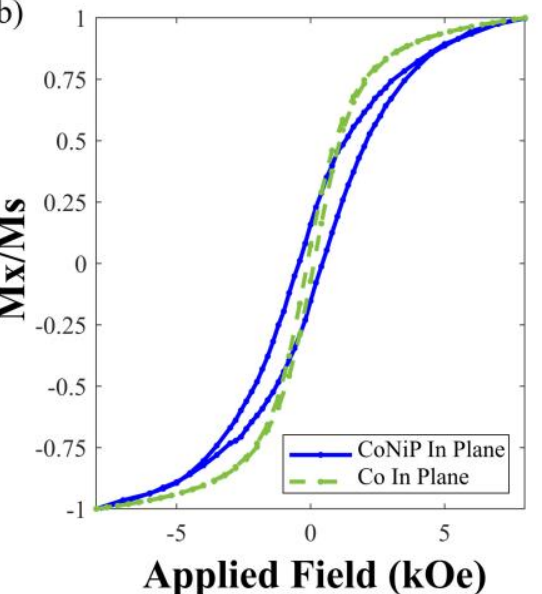

(c)

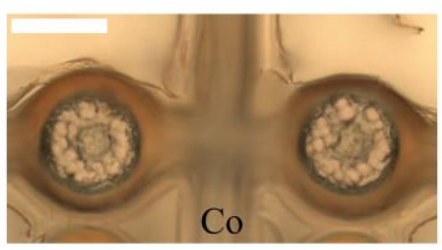

(d)

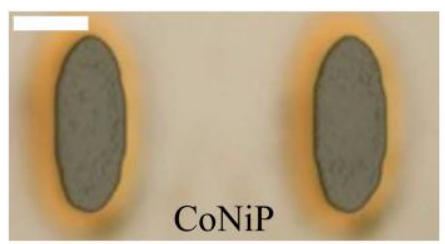

Fig. 2. (a) Dependence of coercivity on the electrodeposition current density for CoNiP films on copper substrates for different temperatures $\left({ }^{\circ} \mathrm{C}\right)$ and $\mathrm{Cr} / \mathrm{Au}$ coated silicon substrates for $30^{\circ} \mathrm{C}$. (b) Normalized VSM hysteresis curves comparing the coercive fields of Co and CoNiP microstructures. Photograph of (c) two Co elements and (d) two CoNiP elements, with a scale bar at the top-left indicating $10 \mu \mathrm{m}$.

choice of electroplating parameters taken from earlier work in our group [16]. For the electrodeposition of the particles a pulsed-electrodeposition technique was used due to the decrease in stress it would have on the smaller shapes [24] Following these methods, circular Co microstructures were produced through electrodeposition at $50^{\circ} \mathrm{C}$ in an aqueous solution of cobalt(II) sulphate heptahydrate $\left(\mathrm{CoSO}_{4} \cdot 7 \mathrm{H}_{2} \mathrm{O}\right)$ mixed at $200 \mathrm{~g} / \mathrm{l}$ with boric acid (40g/l) $(\mathrm{pH} 4)$. Figure 2(b) compares hysteresis curves from arrays of $\mathrm{CoNiP}$ and $\mathrm{Co}$ microstructures grown on gold-coated silicon, while Fig. 2(c) and 2(d) show the electrodeposited elements. The average coercive field for $\mathrm{Co}$ elements was around 120 Oe (electroplated at a current density of $11 \pm 2 \mathrm{mAcm}^{-2}$ ) with the saturation magnetization similar to standard values for polycrystalline cobalt. The in-plane coercivity of the CoNiP microstructures was 435 Oe (produced at $30^{\circ} \mathrm{C}$ with an average current density of $30 \pm 12 \mathrm{mAcm}^{-2}$ ) with an out-ofplane coercivity of 690 Oe. Although this is smaller than the coercive field of CoNiP films, it is still larger that the Co microstructures, which reached only $120 \mathrm{Oe}$.

The measurements were performed on a macroscopic array of elements of the same shape and size, so to be implemented in a further fabrication of membranes (see section IV).

\section{FABRicAtion OF the Elasto-Magnetic MeMbrane}

The next objective in this piece of work is to integrate the magnetic structures in elastic material that could be deformed in the presence of a magnetic field. This means the elastic material has to be structured to provide sufficient elasticity while still having enough volume to encapsulate the magnetic elements. Due to these requirements, the employed fabrication procedure of elasto-magnetic membranes requires an intricate step-by-step process involving several different techniques.
There are five main fabrication sections during the process: substrate preparation, fabrication of hard magnetic elements, fabrication of soft magnetic elements, production of the elastic structure and the liberation of the membrane. The major steps in the fabrication process are illustrated in Fig. 3.

To prepare the substrate for the patterning of the magnetic particles, a $1 \mathrm{~mm}$ thick glass substrate with an area of $24 \mathrm{~mm}$ x $24 \mathrm{~mm}$ was coated with a $600 \mathrm{~nm}$ layer of $950 \mathrm{~K} \mathrm{A6}$ polymethyl methacrylate (PMMA) via spin coating. This was a sacrificial layer that was used in the liberation of the membrane at the end of the fabrication process. The substrate was plated with $100 \mathrm{~nm}$ of aluminum, $5 \mathrm{~nm}$ of chromium and $50 \mathrm{~nm}$ of gold using a thermal evaporator, forming a multilayer of glass/PMMA/Al/Cr/Au [Fig. 3a(i)]. These metal layers were required to produce a conductive layer for the electrodeposition process but needed to be removed prior to membrane liberation in order to enable membrane actuation. Due to the harmful nature of the $\mathrm{Cr}$ and $\mathrm{Au}$ etchants with the CoNiP elements, we decided to partially etch the $\mathrm{Cr}$ and $\mathrm{Au}$ layers before the magnetic particles were produced. This involved using photolithographed patterns that were the inverse of where the particles and contacts would be, enabling electrodeposition to take place without hindering later fabrication steps. To do this, a layer of Shipley S1828 photo resist was spun at $2000 \mathrm{rpm}$ and baked at $120^{\circ} \mathrm{C}$ for $90 \mathrm{~s}$. A

Durham Magneto Optics MicroWriter ML2 was used to expose the inverse pattern onto the resist. Initially, markers were written and developed into the resist in order to align future lithographic steps. Subsequently, the inverse pattern of the particles and contacts for the electrodeposition was written. The resist was developed using the solution MF319 and the exposed $\mathrm{Au}$ and $\mathrm{Cr}$ was removed by etching in standard gold etchant (Sigma-Aldrich) for $4 \mathrm{~s}$ and chromium etchant (Sigma-Aldrich) for $7 \mathrm{~s}$, respectively. After the 


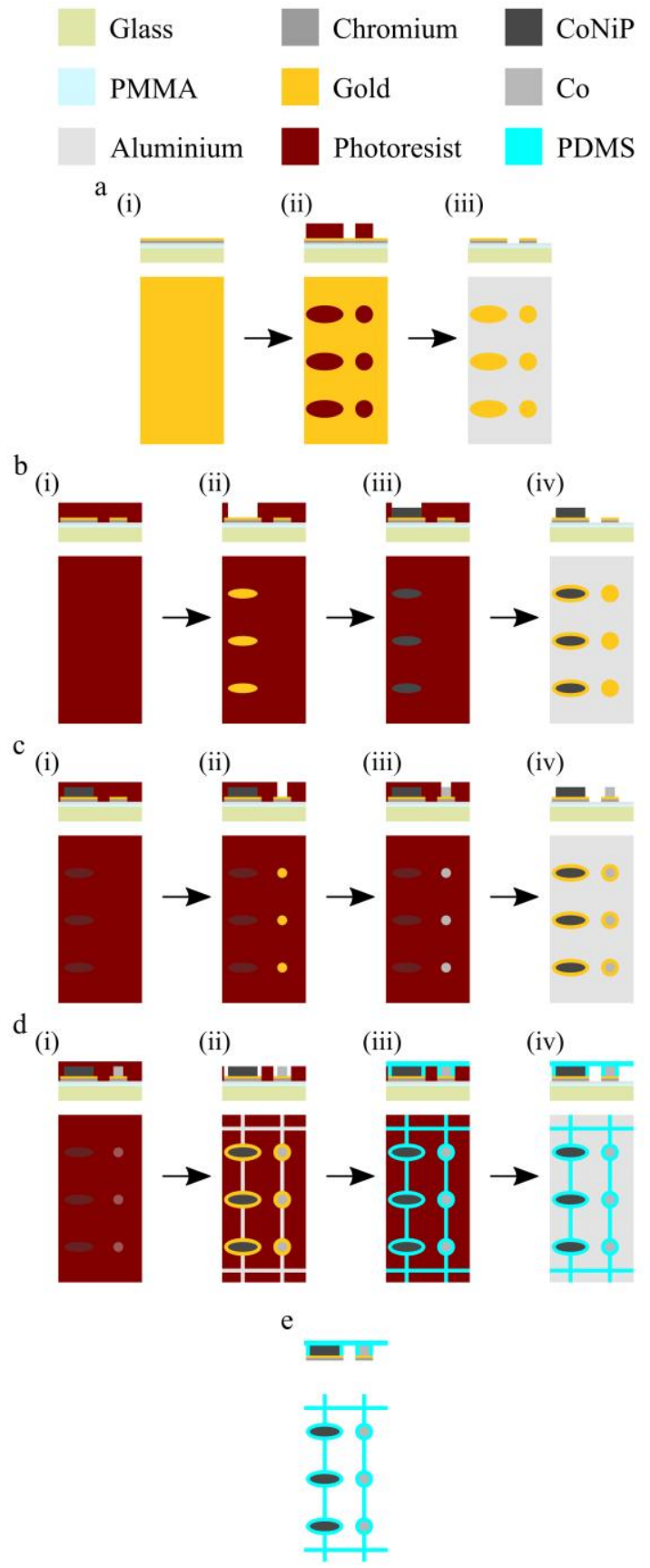

Fig. 3. (a)-(e) Diagram showing the fabrication steps of producing an elastomagnetic membrane. The upmost diagram for each letter shows the cross section with the below photograph showing the view from directly above the sample. (a) Substrate preparation steps. (a-i) Substrate with $600 \mathrm{~nm}$ PMMA spun on followed by $100 \mathrm{~nm}$ of aluminum, $5 \mathrm{~nm}$ of chromium, and 50 $\mathrm{nm}$ of gold layered on top using a thermal evaporator. (a-ii) Lithographic step for the inverse of the magnetic particles, this is for the etching of (a-iii) Au and $\mathrm{Cr}$ with the removal of the resist. (b) Process of producing CoNiP particles. (b-i) Sample when the resist is spun on the sample again and lithograph is used in (b-ii) to create the molds for the hard magnetic particles. (b-iii) Electrodeposition of the hard magnetic particles and (b-iv) when the resist is removed. (c) Similar process for the soft magnetic particles. (c-i) Spinning on resist. (c-ii) Writing the mold and developing. (c-iii) Electrodeposition. (c-vi) Removal of the resist. (d) Production of the links. (di) Resist is added again for the lithographic process of creating the mold for (d-ii) links. (d-iii) When the PDMS is added to the sample to produce these links and the etching of the PDMS to produce the links of an appropriate thickness. (d-iv) Removal of the resist again. (e) Final step the removal of the membrane from the substrate by etching the aluminum and removing the PMMA. removal of the resist with acetone, the substrate remained coated in PMMA and Aluminum with chromium and gold layered features [Fig. 3a(iii)]. Note that the presence of the $\mathrm{Al}$ layer prevented the acetone from removing the sacrificial PMMA layer at this stage.

Patterning of the CoNiP particles was produced with photolithography, however, three layers of photoresist were applied in order to be able to electrodeposit thicker structures (the tri-layered resist was approximately $8 \mu \mathrm{m}$ thick in total), [Fig. 3b(i)]. After the shapes of the CoNiP magnetic elements were written and developed [Fig. 3b(ii)], an oxygen plasma $(50 \mathrm{sccm})$ was used to etch residual resist in the patterned area to ensure the gold surface was clear for electrodeposition. After this process, the sample was prepared for the electrodeposition of $\mathrm{CoNiP}$ (which is discussed in the previous section), this produced $6 \mu \mathrm{m}$ thick particles [Fig. 3b(iii)]. The resist was required to be removed [Fig. 3b(iv)] for the production of the Co particles. Co particles were produced following similar steps [Fig. 3c(i) - (iv)], using the same electrodeposition parameters as the previous section.

The next steps take on the fabrication of the elastic structure. A further three layers of photoresist were spun [Fig. $3 \mathrm{~d}(\mathrm{i})]$ and the link pattern photolithography was performed [Fig. 3d(ii)], which created a mold for the elastic link material. As discussed previously, the links were required to be capable of elastic deformation under magnetic forces, but rigid enough to hold the magnetic elements embedded in it, due to this Sylgard 184 polydimethylsiloxane (PDMS, mixed in a 10:1 ratio of base:curing agent) was selected as the link material. Not only could this be spin-coated to a thickness less than 100 $\mu \mathrm{m}$ but also it has a relatively low Young's moduli and a high Poisson's ratio. The PDMS was spun at $7500 \mathrm{rpm}$ and cured by baking on a hotplate at $115^{\circ} \mathrm{C}$ for 35 minutes. While filling the mold created by the photoresist, it also left excess PDMS on top of the resist, forming a continuous film above the patterned area. To remove the excess PDMS the sample was exposed to $75 \mathrm{~W}, 20$ mTorr inductively coupled plasma (ICP) etch with $\mathrm{SF}_{6}(10 \mathrm{sccm})$ [25] and $\mathrm{O}_{2}(2 \mathrm{sccm})$. To avoid overheating and consequent damage to the sample, the ICP etch was applied in pulses of $30 \mathrm{~s}$, followed by a $30 \mathrm{~s}$ rest, until the PDMS thickness had been reduced to just below the level of the resist, [Fig. 3d(iii)].

After the removal of the resist [Fig. 3d(iv)], the membrane was essentially complete, but remained attached to the substrate. Since MF319 (the photoresist developer) etches aluminum but does not affect the patterned magnets, it was used to remove the Al layer underneath the membrane, exposing the sacrificial layer of PMMA. A thick PDMS frame was attached to the edges of the sample with uncured PDMS, which was then cured in situ to create adhesion. This frame had a $2 \mathrm{~mm}$ hole in the center, which the membrane was attached across after liberation. The sample was then ready for the removal process from the substrate. Liberation of the membrane from the fabrication substrate was achieved by soaking in acetone to dissolve the sacrificial PMMA layer and free the device [Fig. 3e]. At this stage the elasto-magnetic membrane was comprised of a network of $\mathrm{CoNiP}$ and $\mathrm{Co}$ 
(a)

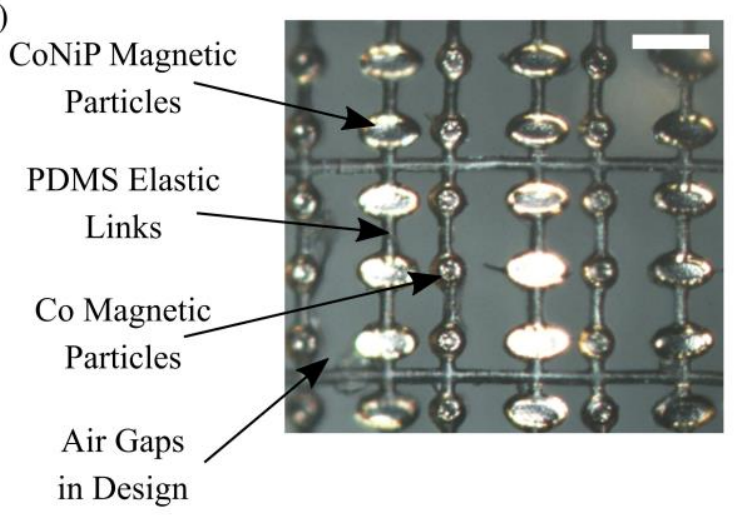

(b)

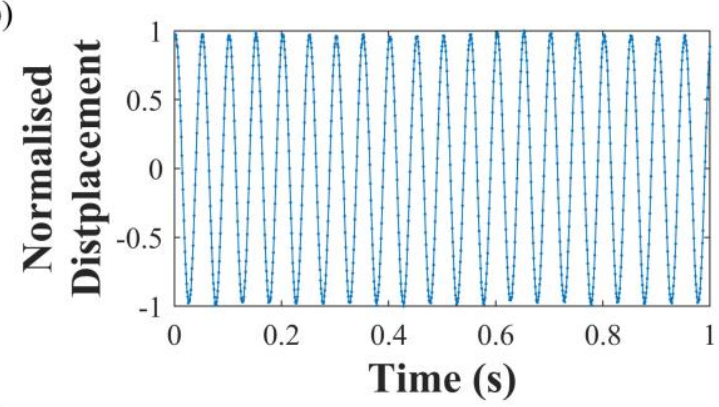

(c)

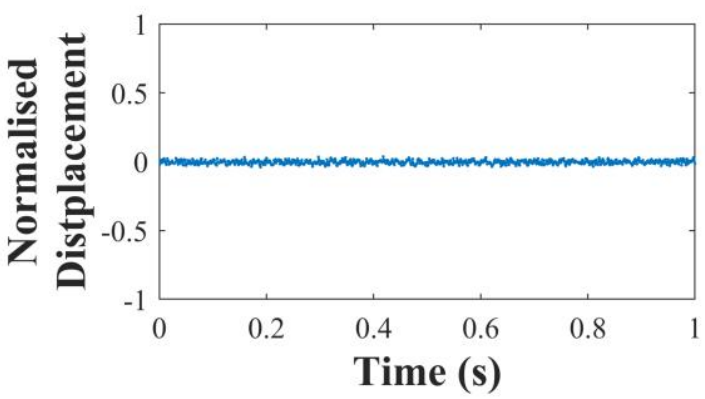

(d)

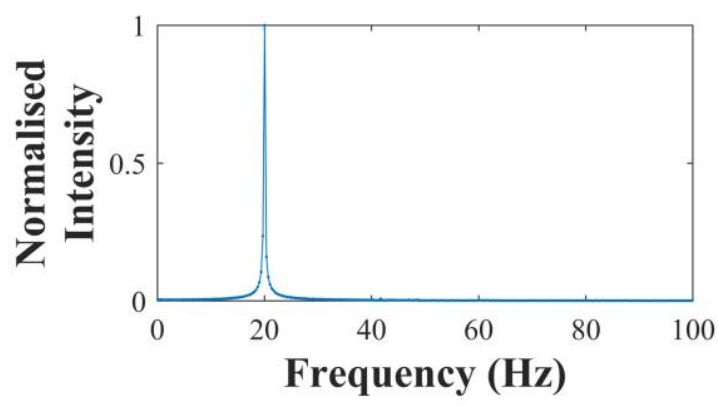

Fig. 4. (a) Photograph of a completed elasto-magnetic membrane indicating the different elements of the membrane with a scale bar at the top- right indicating $100 \mu \mathrm{m}$. The normalized $\mathrm{z}$ displacement against time of the elasto-magnetic membrane (b) in a $200 \mathrm{Oe} 20 \mathrm{~Hz}$ magnetic field and (c) when no magnetic field is present. (d) The Fourier transform of the motion in (b).

particles embedded in PDMS links connecting the particles together, to form a microscale patterned membrane that was attached to a macroscale frame [Fig. 4(a)]. To fix the magnetization direction of the particles, the membrane was saturated in a $1.8 \mathrm{kG}$ field, (in this case using a VSM).

\section{Membrane Actuation}

To test the performance of the elasto-magnetic membrane, it was placed into a microscope system housing a single axis Helmholtz coil pair (Platform Kinetics Ltd). Observing the membrane in air, its behavior was imaged under an oscillating magnetic field. The oscillating magnetic field was tested with a range of frequencies from 1-45 Hz applied on the surface of the membrane. Consistent behavior was observed in this range and although the field was applied in the plane of the membrane surface, it produced an out-of-plane actuation, such that the membrane moved in and out of focus. The oscillations of the particles were assessed optically to estimate the mechanical displacements by measuring the reflected intensity from the membrane. Figure 4(b) shows a trace of when the magnetic field (200 G $20 \mathrm{~Hz}$ ) was applied compared to when there was no applied field [Fig. 4(c)]. The frequency of oscillation of the membrane matches that of the applied field [Fig. 4(d)]. It was found that for a given amplitude of the applied field $(200 \mathrm{G})$ at $20 \mathrm{~Hz}$, the rotation of the particles were reaching $15^{\circ}$, with the main degree of motion perpendicular to the surface of the membrane.

\section{CONCLUSION}

To conclude, this work has successfully shown how ferromagnetic elements can be incorporated into a microscale elastic structure. This was achieved via a multistage process with a number of photolithographic steps, as well as electrodeposition and PDMS etching. We have shown that microscale CoNiP elements can be produced via electrodeposition to be suitably hard for MEMS applications, with coercive fields of 435 Oe. As a proof-of-principle demonstration, the membrane was shown to deform in the presence of an alternating magnetic field. While future challenges will focus on establishing the correct geometry and actuation for particular applications, this work has addressed major difficulties in device fabrication and establishes a strong basis for research in this area.

All data created during this research are openly available from the University of Exeter's institutional repository [26].

\section{ACKNOWLEDGMENT}

This project has received funding via the EPSRC Centre for Doctoral Training in Metamaterials (Grant No. EP/L015331/1) as well as from the European Union's Horizon 2020 research and innovation program under grant agreement No. 665440. Authors would also like to acknowledge contribution by Platform Kinetics Ltd. For providing the magneto-optic equipment for activation and observation of the membrane mechanics as well as optimization of the setup and useful discussions about the measurements.

\section{REFERENCES} ( LDW ) of magnetic structures," AIP Adv., vol. 8, no. 5, p. 56322, 2018.

[2] D. P. Arnold and N. Wang, "Permanent magnets for MEMS," $J$. 

Y. Li, J. Kim, M. Kim, A. Armutlulu, and M. G. Allen, "Thick Multilayered Micromachined Permanent Magnets With Preserved Magnetic Properties," J. Microelectromechanical Syst., vol. 25, no. https://ore.exeter.ac.uk/repository/.

[4] Y. Xuetao, W. Yu, S. Dongbai, and Y. Hongying, "Influence of pulse parameters on the microstructure and microhardness of nickel electrodeposits," Surf. Coatings Technol., vol. 202, no. 9, pp. 18951903, 2008.

[5] E. M. Purcell, "Life at low Reynolds number," Am. J. Phys., vol. 45, no. 1, pp. 3-11, 1977.

[6] J. D. Zahn, "Micropump Applications in Bio-MEMs," in Bio-MEMS - Technologies and Applications, W. Wang and S. A. Soper, Eds. CRC Press, 2007, pp. 142-176.

[7] L. Zhu and H. A. Stone, "Rotation of a low-Reynolds-number watermill : theory and simulations," J. Fluid Mech., vol. 849, pp. 57-75, 2018.

[8] O. Raz and J. E. Avron, "Swimming, pumping and gliding at low Reynolds numbers," New J. Phys., vol. 9, p. 437, 2007.

[9] A. K. Au, H. Lai, B. R. Utela, and A. Folch, "Microvalves and Micropumps for BioMEMS," Micromachines, vol. 2, no. 2, pp. 179-220, 2011.

[10] K. S. Ryu, K. Shaikh, E. Goluch, Z. Fan, and C. Liu, "Micro magnetic stir-bar mixer integrated with parylene microfluidic channels," Lab Chip, vol. 4, no. 6, pp. 608-613, 2004.

[11] T. Sawetzki, S. Rahmouni, C. Bechinger, and D. W. M. Marr, "In situ assembly of linked geometrically coupled microdevices," Proc. Natl. Acad. Sci., vol. 105, no. 51, pp. 20141-20145, 2008.

[12] F. Y. Ogrin, P. G. Petrov, and C. P. Winlove, "Ferromagnetic microswimmers," Phys. Rev. Lett., vol. 100, no. 21, pp. 21812 21816, 2008.

[13] A. D. Gilbert, F. Y. Ogrin, P. G. Petrov, and C. P. Winlove, "Theory of ferromagnetic microswimmers," Q. J. Mech. Appl. Math., vol. 64, no. 3, pp. 239-263, 2011.

[14] J. K. Hamilton, M. T. Bryan, A. D. Gilbert, F. Y. Ogrin, and T. O. Myers, "A new class of magnetically actuated pumps and valves for microfluidic applications," Sci. Rep., vol. 8, no. 933, pp. 1-12, 2018.

[15] J. K. Hamilton, P. G. Petrov, C. P. Winlove, A. D. Gilbert, M. T. Bryan, and F. Y. Ogrin, "Magnetically controlled ferromagnetic swimmers," Sci. Rep., vol. 7, p. 44142, 2017.

[16] S. M. Weekes, F. Y. Ogrin, and W. A. Murray, "Fabrication of Large-Area Ferromagnetic Arrays Using Etched Nanosphere Lithography," Langmuir, vol. 20, no. 4, pp. 11208-11212, 2004.

[17] P. Cojocaru, L. Magagnin, E. Gomez, and E. Valles, "Electrodeposition of CoNiP films with modulated magnetic behaviour," Trans. Inst. Met. Finish., vol. 89, no. 4, pp. 194-197, 2011.

[18] K. Chouarbi, M. Woytasik, E. Lefeuvre, and J. Moulin, "SmCo micromolding in an aqueous electrolyte," Microsyst. Technol., vol. 19, no. 6, pp. 887-893, 2013.

[19] A. Walther, C. Marcoux, B. Desloges, R. Grechishkin, D. Givord, and N. M. Dempsey, "Micro-patterning of NdFeB and SmCo magnet films for integration into," J. Magn. Magn. Mater., vol. 321, pp. 590-594, 2009.

[20] F. Luborsky, "High coercive force films of cobalt-nickel with additions of group VA and VIB elements," IEEE Trans. Magn., vol. 6, no. 3, pp. 502-506, 1970.

[21] C. Wu et al., "Tuning microstructure and magnetic properties of electrodeposited CoNiP films by high magnetic fi eld annealing," $J$. Magn. Magn. Mater., vol. 416, pp. 61-65, 2016.

[22] X. He et al., "Structure and magnetic properties of CoNiP nanowire arrays embedded in AAO template," J. Cryst. Growth, vol. 310, no. 15, pp. 3579-3583, 2008.

[23] M. D. Cooke, D. A. Allwood, D. Atkinson, G. Xiong, C. C. Faulkner, and R. P. Cowburn, "Thin single layer materials for device application," J. Magn. Magn. Mater., vol. 257, pp. 387-396, 2003.

[24] M. S. Chandrasekar and M. Pushpavanam, "Pulse and pulse reverse plating-Conceptual, advantages and applications," Electrochim. Acta, vol. 53, no. 8, pp. 3313-3322, 2008.

[25] M. P. Anenden, M. Svehla, N. H. Lovell, and G. J. Suaning, "Process Development for Dry Etching Polydimethylsiloxane for Neural Electrodes," in 33rd Annual International Conference of the IEEE EMBS, 2011, pp. 2977-2980. 